\title{
LA FORMACIÓN DOCTORAL EN ADMINISTRACIÓN EN COLOMBIA: REFLEXIÓN FENOMENOLÓGICA DE UN INVESTIGADOR*
}

\author{
Luis Antonio Orozco**
}

* doi: 10.11144/Javeriana.cao29-52.fdac. Este artículo es producto de “La investigación en administración en América Latina", financiado por la Universidad Externado de Colombia, desde enero de 2009 a la fecha. El autor agradece a su esposa Jaidy Buitrago por la revisión de este artículo, así como también a Carlos Restrepo de la Universidad Externado de Colombia. El artículo se recibió 03/04/2016 y se aprobó el 30/05/2016. Sugerencia de citación: Orozco, L. A. (2016). La formación doctoral en administración en Colombia: reflexión fenomenológica de un investigador. Cuadernos de Administración, 29 (52), 115-139. http://dx.doi.org/10.11144/ Javeriana.cao29-52.fdac.

** Doctor en Administración de la Universidad de los Andes, Bogotá, Colombia, 2013. Profesor investigador de la Facultad de Administración de Empresas de la Universidad Externado de Colombia. Es investigador adjunto del Science, Technology \& Policy Studies (STEPS) de la Universidad de Twente, Países Bajos, y del National Center for Digital Government de la Universidad de Massachusetts, Estados Unidos.

Correo electrónico: luis.orozco@uexternado.edu.co 


\section{La formación doctoral en administración en \\ Colombia: reflexión fenomenológica de un investigador}

\section{RESUMEN}

En el marco de la epistemología de la fenomenología trascendental de Husserl, este artículo presenta la reflexión de uno de los primeros doctores graduados en administración en Colombia. Desde este caso se pretende ilustrar cómo se ha reproducido una comunidad científica en administración en un entorno institucional que promueve la colaboración científica particularmente en grupos de investigación. Se busca aportar tanto a los administradores de la ciencia como a los nuevos doctorandos las características de un proceso de formación doctoral, su incidencia en las dinámicas de construcción de redes, grupos de investigación y generación de nuevo conocimiento.

Palabras clave: administración, formación doctoral, Colombia, fenomenología trascendental, administración de la investigación.

Clasificación JEL: M11, A23
Doctoral training in management in Colombia: A researcher's phenomenological reflection

\section{A formação doutoral em administração na \\ Colômbia: reflexão fenomenológica de um pesquisador}

\section{ABSTRACT}

Within Husserl's epistemological framework of transcendental phenomenology, this article presents the reflection of one of the first doctors graduated in management in Colombia. From this case it pretends to illustrate how a scientific community in management has reproduced in an institutional environment that promotes permanent scientific collaboration in research groups. It aims to contribute to both science managers and new doctorate students the characteristics of a doctoral training process, its incidence in the dynamics of network construction, research groups and creation of new knowledge.

Keywords: management, doctoral training, Colombia, transcendental phenomenology, research management.

JEL Classification: $\mathrm{M}_{11}, \mathrm{~A}_{23}$

\section{Resumo}

No âmbito da epistemologia da fenomenologia transcendental de Husserl, este artigo apresenta a reflexão de um dos primeiros doutores graduados em administração na Colômbia. A partir deste caso, pretende-se ilustrar como se reproduziu uma comunidade científica em administração num meio institucional que promove a colaboração científica particularmente em grupos de pesquisa. Procura-se fornecer tanto aos administradores da ciência quanto aos novos doutorandos as características de um processo de formação doutoral, sua incidência nas dinâmicas de construção de redes, grupos de pesquisa e geração de novo conhecimento.

Keywords: administração, formação doutoral, Colômbia, fenomenologia transcendental, administração da pesquisa.

Classificação JEL: M11, A23 


\section{Introducción}

Los estudios sobre el desarrollo de la investigación en administración en Colombia inician con los trabajos del profesor Carlos Dávila de la Universidad de los Andes - Uniandes en la década de 1980 y principios de 1990 (Dávila, 1980, 1986, 1989, 1991; Rodríguez, Dávila y Romero, 1992; Dávila y Samper, 1994), revitalizados con investigación empírica del profesor Florentino Malaver de la Pontificia Universidad Javeriana (Malaver, 2000, 2006 y Malaver et al., 2000) y el profesor Gregorio Calderón de la Universidad Nacional de Colombia (Calderón y Castaño, 2005 y Calderón et al., 2010).

Dávila $(1986,1989)$ propuso la necesidad de formar doctores en el país con las más altas calidades de la investigación científica internacional, capaces de asumir el reto de producir teorías que correspondieran a las realidades latinoamericanas. Con la reestructuración de Colciencias y la organización del Sistema Nacional de Ciencia y Tecnología (SNCyT) en la década de 1990, se dio un avance significativo en la generación de capacidades en investigación, en el que la formación doctoral jugaría un papel central (Villaveces, 1991, 2003).

El reporte sobre la investigación en administración de Rodríguez, Dávila y Romero (1992), realizado en el marco de los estudios que fundamentaron la construcción de la política del nuevo SNCyT, arrojó un panorama desalentador sobre la capacidad de producción científica. Los autores argumentan que la producción científica era de baja calidad, de carácter local y de modesta circulación, y que "solo un ínfimo número de artículos (6) ha tenido circulación internacional" (ibíd., p. 67). Las pocas revistas en administración editadas en Colombia presentaban "deficiencias en su periodicidad, poco rigor en la selección de artículos, predominio de ensayos y escasez de informes de investigación" (Rodríguez et al., 1992, pp. 70-71).

Lo anterior se ratificaba con el estudio de Malaver (2000) sobre 416 publicaciones entre 1965 y 1998, que encontró una investigación en administración concentrada en cuatro universidades ${ }^{1}$ y poco difundida, especialmente a nivel internacional. En las revistas nacionales "apenas se publica un artículo derivado de investigación por cada dos ejemplares" (ibíd., p. 65), y advirtió que pese al vertiginoso incremento de posgrados en

1 Universidad de los Andes, Universidad Nacional de Colombia, Universidad del Valle y Pontificia Universidad Javeriana. 
administración, no existían las bases investigativas para una educación de calidad, especialmente porque en las escuelas de administración la investigación era misionalmente marginal (ibíd., p. 67).

Dávila y Malaver (2004) entienden que la maduración de un entorno institucional para la investigación en Colombia, apoyado en una política de grupos de Colciencias que anima la colaboración científica, ha tenido impactos positivos en la creación de capacidades para la investigación en administración. Los autores consideran que se han generado dinámicas importantes en la creación de nuevo conocimiento que deben madurarse en la publicación internacional de alta calidad. Reconocen que aún queda mucho por avanzar, ya que de las 118 facultades de administración solo once tienen grupos de investigación reconocidos por su producción intelectual.

Malaver (2006) realizó un estudio que tituló “El despegue de la investigación colombiana en administración"2 ${ }^{2}$ en el que describió el desarrollo de capacidades, especialmente en la realización colectiva de la investigación, y el apoyo de la política científica y tecnológica en la institucionalización de la investigación y sus mecanismos de comunicación. Afirma Malaver que entre 2000 y 2006 :

[S]e presentó un significativo proceso de internacionalización en la divulgación del conocimiento generado a través de la investigación. Esto sugiere un franco proceso de superación del aislamiento internacional existente a finales de la década de los noventa. Por lo tanto, junto al despegue de la investigación y el aumento en la cantidad y calidad de los productos académicos obtenidos, el área está en un promisorio proceso de internacionalización en la divulgación de su producción (Malaver, 2006, p. 106).

Las estadísticas del Observatorio Colombiano de Ciencia y Tecnología muestran que la producción de autores de instituciones colombianas en revistas indexadas en Scopus para administración, negocios y contaduría entre 2003 y 2012, ha tenido un incremento importante que da evidencia del despegue de la investigación de calidad en administración como se muestra en la tabla 1.

2 Apoyado en la información que le suministré siendo joven investigador de Colciencias en el Observatorio Colombiano de Ciencia y Tecnología en 2004. 


\section{Tabla 1}

Producción en revistas de administración, negocios y contaduría entre 2003 y 2012 en Scopus

\begin{tabular}{lcccccccccc}
\hline \multicolumn{1}{c}{ Año } & 2003 & 2004 & 2005 & 2006 & 2007 & 2008 & 2009 & 2010 & 2011 & 2012 \\
\hline Artículos & 7 & 7 & 16 & 16 & 28 & 66 & 93 & 85 & 112 & 135 \\
Economía y finanzas & 15 & 11 & 18 & 15 & 46 & 82 & 118 & 123 & 135 & 146 \\
\hline
\end{tabular}

Fuente: ОСут (2013, p. 128).

Finalmente el trabajo del grupo de investigación de Calderón et al. (2010), presenta un balance de la investigación en administración en Colombia a partir de una amplia investigación cualitativa en las facultades del país, con entrevistas a profundidad, que revela los estímulos y las restricciones institucionales para el desarrollo científico del campo, así como los progresos y las limitaciones en el avance de la producción de conocimiento en general.

Al evaluar la percepción de los decanos sobre la participación de las facultades en programas de fomento a la investigación externos a la universidad, Calderón et al. (2010 citado en Orozco, 2015, pp. 120-121) muestran que si bien existen recursos para destinar a la investigación, existe una preocupación por la escaza comunidad científica en las facultades para desarrollar un sistema de investigación, donde pares evaluadores permitan mejorar la capacidad de crear nuevo conocimiento de impacto nacional e internacional.

De acuerdo con Calderón et al. (2010) las facultades de administración participan poco en programas como el de jóvenes investigadores de Colciencias, no son activas en hacer intercambios de investigadores, poco promueven el involucramiento de los investigadores con las empresas y su participación en misiones tecnológicas y programas de investigación es marginal. “Esto lleva a evidenciar que en lo único que se ha avanzado, sin ser suficiente, es en la formación de doctores o capacitaciones internacionales de investigadores" (ibíd., p. 133). Calderón y su grupo también encuentran en sus entrevistas que "los desarrollos en la investigación en administración se deben a procesos y actividades individuales, y no a una actividad grupal dirigida por un ente institucional" (ibíd., p. 130 citado en Orozco, 2015, p. 121).

Con el ánimo de complementar los estudios sobre la investigación en administración en Colombia y América Latina, enfoqué mi tesis doctoral al desarrollo de una explicación alternativa sobre el incremento de la producción científica de alta calidad internacional en el campo, más allá del número de doctores e incentivos institucionales a partir del análisis de la colaboración científica entre individuos y organizaciones. Estudiando las 
publicaciones de las escuelas de administración escalafonadas por América Economía entre 2008 y 2011, pude generar proposiciones sobre la incidencia de la diversidad entre personas y heterogeneidad entre organizaciones en la formación de capacidades para la producción de nuevo conocimiento (Orozco, 2015).

Las ideas normativas que racionalmente justifican las políticas de investigación indican que la formación doctoral y la creación de redes de colaboración científica, que se materializan en grupos de investigación y otras formas de trabajo colectivo, conducen a una mayor producción científica internacional. Como estudioso de los grupos de investigación en Colombia tenía evidencia de la importancia de los doctores y las redes en el desempeño científico (Orozco y Chavarro, 2005; Orozco, Ruiz, Bonilla y Chavarro, 2013; Ruiz et al., Chavarro, Villaveces y Orozco, 2008). Sin embargo, Gantman (2008) encuentra que el número de doctores en las escuelas de administración escalafonadas por América Economía para América Latina no presentan una relación positiva con la producción internacional en revistas indexadas en el ISI Web of Science, resultado que ratifico con mi tesis doctoral (Orozco, 2015; Orozco y Villaveces, 2015).

Lo anterior plantea el problema de investigación de este artículo, ya que no es claro cómo los doctores y en particular la formación doctoral contribuyen a la generación de nuevo conocimiento evidenciado en la publicación científica de alta calidad, en un proceso de construcción y participación en redes de colaboración científica animadas por políticas e incentivos para la investigación. Así resulta la pregunta de investigación que guía este artículo: ¿Cuál es el rol de la colaboración científica y las políticas de investigación en el proceso de formación doctoral para impulsar la producción científica de alta calidad?

El artículo está dividido en tres secciones. En la primera presento el enfoque epistemológico de la fenomenología trascendental de Husserl y algunos trabajos reflexivos sobre la formación doctoral en administración. En la segunda parte desarrollo una reflexión guiada por preguntas que me obligan a exponer una descripción consciente sobre mi proceso de formación doctoral. En la tercera parte expongo unas reflexiones sobre la formación doctoral en Colombia y algunas consideraciones sobre la estructura, los incentivos y la colaboración científica en el campo de la administración.

\section{Epistemología de la fenomenología trascendental de Husserl}

Husserl (1949) propone que existe un pensamiento natural que deviene de nuestros actos comunes y prácticos de la experiencia vivencial. La realidad es construida por quien vive 
el fenómeno, y está imbricada en la interpretación que hace sobre su experiencia. El investigador es un individuo activo, con identidad e intereses, con los que da explicaciones sobre el mundo fenomenológico a partir de su consciencia reflexiva que es traducida en su discurso descriptivo. De esta forma, el conocimiento se construye, a diferencia del positivismo, en un acto que expresa el entendimiento y el sentido del investigador que experimenta una realidad. La forma como el investigador percibe el mundo está cargado de una intencionalidad y unas emociones que no se pueden separar del acto cognoscitivo. De esta forma, la epistemología de la fenomenología transcendental define un método para que, a través del discurso reflexivo y subjetivo, el investigador reexamine su experiencia de forma consciente para describir, en un proceso interpretativo, su concepción de la realidad sin separar la mente de los objetos.

Existen revistas como el International Journal of Qualitative Methods en las que se encuentran serias reflexiones y proposiciones sobre el método de investigación fenomenológica, y se discuten diferentes vías de creación de conocimiento como la propuesta de Husserl y su método de variaciones libres con la que el investigador puede describir un fenómeno (Laverty, 2003, p. 23). Para desarrollar este estudio adopto una metodología en la que describo mi experiencia y tomo distancia de ella a partir de preguntas que formulo para mi autoreflexión en función de develar aspectos de los que logro consciencia sobre mi proceso de formación doctoral.

En la literatura existen pocos trabajos que desde una perspectiva reflexiva y hermenéutica describan el proceso de formación doctoral en administración. El artículo de Mishra (2015) describe la forma en la que el autor decide entrar a un programa de doctorado en administración, selecciona un tema de investigación y una metodología para su tesis, finalizando con una serie de reflexiones sobre los elementos que le permitieron graduarse, entre ellos el manejo del tiempo, de las situaciones familiares y de la organización de un trabajo disciplinado en torno a una pregunta de investigación.

También se encuentra en el libro Great Minds in Management, editado por Ken Smith y Michael Hitt en 2005, la descripción reflexiva y fenomenológica de los autores contemporáneos más destacados en administración sobre la forma en la que hicieron su doctorado, desarrollaron su carrera investigativa y propusieron sus teorías. Sin embargo, fuera de la literatura anglosajona no encontré una descripción del proceso de formación doctoral en administración. Por lo tanto este artículo es un aporte en este sentido. 


\section{El doctorado en administración en la Universidad de los Andes, Colombia}

El programa de doctorado en administración empezó a estructurarse a inicios de la década de 1990 con Carlos Dávila y Enrique Ogliastri, cuando era decano Jorge Hernán Cárdenas, gran promotor de la formación doctoral en Colombia (Cárdenas, 1991). La Universidad de los Andes (Uniandes) estableció un acuerdo con la Universidad de Tulane en Estados Unidos, para la formación doctoral de sus profesores. También enfocó programas de investigación en temas como historia empresarial y comportamiento organizacional para crear capacidades de formación doctoral. En 2006 el Ministerio de Educación Nacional dio el registro calificado al programa para iniciar en 2007.

\subsection{Antecedentes de la vinculación al doctorado en administración}

Cursé la carrera profesional en administración de empresas en la Pontificia Universidad Javeriana entre 1998 y 2002. Mi profesor de gestión tecnológica, Florentino Malaver, me postuló en el Observatorio Colombiano de Ciencia y Tecnología (OCyT) en 2002 para hacer mi práctica profesional ${ }^{3}$. En 2003 me hice becario de Colciencias, la agencia pública encargada de la política de ciencia, tecnología e innovación en Colombia, como joven investigador bajo la tutela del director del OCyT, el químico José Luis Villaveces. Me había graduado como administrador de la Javeriana con un plan de negocios que se materializó y feneció en el 2003. La experiencia de ese emprendimiento la publiqué en mi primer artículo académico en la revista Estudios Gerenciales (Orozco y Chavarro, 2005). Trabajé en el OCyT viviendo una experiencia grata como miembro de un grupo de investigación muy diverso y productivo, donde participé en redes heterogéneas con entidades como la OEA, DNP, FAO y Colciencias. En ese tiempo inicié mis investigaciones sobre las redes de colaboración científica (Orozco y Chavarro, 2006) y el impacto de la ciencia en la sociedad (Villaveces, Orozco, Chavarro, Olaya y Suárez, 2005).

En enero de 2006, luego de regresar de una pasantía en la Red Iberoamericana de Indicadores de Ciencia y Tecnología (RICyT) en Buenos Aires, me vinculé a Uniandes para continuar trabajando con Villaveces, quien había asumido el cargo de director de investigaciones de Uniandes, y tener la oportunidad de hacer parte de la primera cohorte de doctorandos de administración. Me animó también el profesor Clemente Forero, con quien

3 Inicié depurando bases de datos, trabajando en el naciente sistema ScienTi Colombia, del que no tenía la dimensión de lo que estábamos forjando. 
había colaborado en un proyecto de investigación, así como la posibilidad de interactuar con el profesor Carlos Dávila.

En 2006 iniciaron los dos primeros programas de doctorado en administración en Colombia, uno en la Universidad EAFIT, pionera en la formación en administración con el primer programa profesional en el campo en 1960, con un enfoque cualitativo importante. El segundo en la Universidad Nacional de Colombia, que tenía un convenio con una universidad en Francia, pero que a la postre tuvo grandes dificultades.

Luego de un riguroso proceso de selección fui admitido en el programa de doctorado en administración de Uniandes. Me otorgaron un crédito para el primer año con la esperanza de que obtuviera financiación del programa de doctorados nacionales de Colciencias, tal como sucedió. En 2007 Colciencias otorgó 50 créditos condonables en el país para todas las disciplinas, y yo fui favorecido con tres años de financiación.

\subsection{El inicio del doctorado}

Yo era el más joven de los estudiantes y el único sin maestría. Me encontré con compañeros de mucho bagaje académico, con experiencia en consultoría, que conocían bien la vida universitaria y el campo de la administración. Tenía para entonces dos artículos en evaluación en revistas internacionales indexadas en el ISI, que finalmente fueron publicados durante el primer semestre de 2007 (Orozco et al. , 2007; Schuler y Orozco, 2007), lo que me valió el respeto de mis compañeros y de algunos profesores. El primer semestre fue reto inmenso: varias veces pensé en retirarme dado el estrés que me causaba la carga académica de mis seminarios doctorales. Estos seminarios, que describo a continuación, tenían un gran volumen de lecturas y era difícil cumplir con las tareas, particularmente con el trabajo final que consistía en la elaboración de un artículo publicable.

La Facultad, a través de la directora del doctorado en esa época, Elvira Salgado, nos animaba a realizar trabajos publicables. Asimismo, las políticas de investigación de la Facultad definían incentivos económicos por la publicación y la citación, tanto en la condonación del crédito que asumimos tres estudiantes para el primer año, como en bonificaciones monetarias, lo que motivaba aún más el esfuerzo por la investigación de calidad. Si bien existían grupos registrados en Colciencias donde los estudiantes doctorales éramos vinculados, y la Facultad esperaba de los grupos coautorías y colaboración entre doctorandos y profesores, yo busqué apoyo en mi grupo informal con Villaveces, y mis amigos Diego Chavarro, literato de la Universidad Nacional de Colombia (UNAL) 
y Cristhian Ruiz, ingeniero ambiental, entonces profesionales de la Vicerrectoría de investigaciones de Uniandes con y Ricardo Bonilla, físico y estudiante del doctorado en ingeniería de Uniandes.

El seminario de epistemología, dirigido por el filósofo Luis Enrique Orozco, era en el que mejor me acomodaba. Ya había trabajado varios de los autores que se debían estudiar en el curso y tenía una notable ventaja frente a mis compañeros. Orozco me reiteró que esperaba que mi trabajo final se publicara en una revista indexada. Lo envié a la Revista de Estudios Sociales, editada en Uniandes, y que recién se había indexado en ISI. Me rechazaron el artículo por no tener una visión crítica sobre la temática tratada: la institución de la ciencia desde la obra de Merton. Le pedía Diego Chavarro, con quien había mantenido un constate diálogo sobre este trabajo, que lo complementáramos para publicarlo juntos. Finalmente el artículo fue aceptado casi tres años después (Orozco y Chavarro, 2010) ${ }^{4}$.

Recibí también la cátedra de teoría organizacional, dictada por el doctor Clemente Forero, ex director de Colciencias y académico de mucho rigor desde el ala economicista de la administración, y el doctor Camilo Dávila, hermano de Carlos Dávila, orientado por la sociología en el análisis organizacional. Fue un curso intenso, de mucho debate, con temas que jamás había tratado antes. El trabajo final lo enfoqué a la comparación, desde la teoría de costos de transacción y la teoría del institucionalismo organizacional, de dos casos de redes promovidas por Colciencias en biotecnología. De nuevo, colaborando con mi grupo informal, el trabajo se publicó cuatro años después en las memorias de un congreso de investigación en administración (Orozco, Chavarro y Ruiz, 2011).

Tomé el seminario de fundamentos de administración y gestión del profesor Carlos Dávila. Su exigencia académica, su rigor en las lecturas, su crítica sobre mis escritos, hicieron que mi esfuerzo se redoblara para cumplir con sus expectativas. Dávila también pedía que los trabajos finales de su seminario resultaran en publicaciones indexadas, como finalmente pude lograrlo, con mi revisión de la obra de Peter Drucker, en la revista Innovar Journal de la UNAL y que también había sido aceptada en el ISI recientemente (Orozco, 2009).

Finalmente cursé un seminario de estadística matemática, donde entendí que mis conocimientos previos eran muy precarios. El primer parcial lo perdí con 1,5/5,0. Esto arruinaba

4 Este artículo no sólo ha sido citado varias veces, sino que incluso fue plagiado por un investigador relativamente conocido en Argentina, el profesor Osvaldo Barsky. Los detalles los puedo suministrar por comunicación personal. 
todo mi esfuerzo en las demás asignaturas. Me dediqué a estudiar matemática, a resolver todos los ejercicios posibles, con la presión de la nota y las implicaciones de perder un curso: la exclusión del programa. Superé el segundo examen con 3,5 y luego de un profundo trabajo con mis compañeros doctorales (la mayoría íbamos perdiendo el curso), y particularmente con el apoyo de mi amigo y colega doctoral, Hernán Herrera, dedicados fines de semana a resolver problemas, pasé mi examen final con 4,0. Este seminario continuó con estadística multivariada en el periodo intersemestral, alternado con el curso de estrategia, en el que me enfoqué a investigar sobre gestión del conocimiento para un artículo que resultaría publicado en una revista nacional indexada casi de forma inmediata (Orozco, Chavarro y Rivera, 2007). Esto de alguna manera me indicaba que iba mejorando en mi capacidad de producir artículos académicos de calidad en colaboración científica.

En el segundo semestre de 2007 tuve un excelente seminario sobre comportamiento organizacional dirigido por Elvira Salgado. Ella aceptó que mi trabajo final, que consistía en una revisión bibliográfica sobre la colaboración para la innovación, se acoplara con el trabajo final del seminario de métodos de investigación y lo extendiera en el seminario de innovación, dirigido por Clemente Forero en el primer semestre de 2008. Apoyado en la segunda encuesta nacional de innovación y desarrollo tecnológico, busqué la forma de estudiar el rol de los doctores en la innovación de la industria manufacturera del país desde el comportamiento organizacional en una perspectiva multinivel. Tanto Elvira como Clemente, a quien presentaba mis avances, me apoyaron en la investigación, pero tuve choques muy fuertes con la profesora de métodos de investigación.

El profesor Villaveces me indicó que sería bueno mostrarle mis avances a Fernando Chaparro ${ }^{5}$ y Jorge Hernán Cárdenas, quienes estaban realizando una investigación sobre los doctorados en el país. Una tarde el profesor Clemente Forero me pidió atender a una periodista de la Revista Semana para contarle mis resultados. No solo salí entrevistado en un artículo sobre educación superior en esa revista ${ }^{6}$, sino que a la postre mi artículo fue publicado por la mejor revista nacional en administración, indexada en el ISI, gracias de nuevo a la colaboración científica con mi grupo de investigación informal (Orozco, Ruiz y Chavarro, 2010). Esto me demuestra la importancia de la fortaleza de los enlaces débiles teorizados por Granovetter (1973).

5 Ex director de Colciencias y promotor de la creación del Sistema Nacional de Innovación en 1994.

6 Ver Revista Semana, n. 1325, 31 de marzo al 7 de abril de 2008, pp. 72-73. 
En el seminario de emprendimiento con el profesor Carlos Dávila recibí un conocimiento de base muy valioso, el cual aproveché para hacer una revisión bibliográfica sobre un tema que veníamos discutiendo con Villaveces: el emprendimiento universitario. Fui invitado por la Universidad Santo Tomás a exponer mi trabajo, que se publicó en su revista de investigaciones (Orozco y Chavarro, 2010). Como se puede leer en mis trabajos con José Luis Villaveces, esa semilla germinó en un estudio sobre la innovación, la transferencia de tecnología y el emprendimiento financiado por CINDA, RedEmprendia y Universia en 2015 (Villaveces y Orozco, 2015), por la cual fui de nuevo entrevistado por Revista Semana ${ }^{7}$.

En el primer semestre de 2008 cursé el seminario de comportamiento organizacional II, dirigido por varios profesores, y con el que pude decantar mejor mi trabajo previo sobre el comportamiento organizacional en innovación. Asimismo el seminario de innovación de Clemente Forero me permitió asentar empíricamente mis avances para lograr la publicación del artículo en una revista ISI como comenté anteriormente (Orozco et al. , 2010).

Finalmente el seminario de teoría organizacional II, dirigido por el profesor Eduardo Wills, me dio la oportunidad de hacer mi trabajo combinando los enfoques de los estudios organizacionales con los estudios sociales de la ciencia y la tecnología, que ya venía avanzando, para mejorar el entendimiento sobre las redes de colaboración científica. El trabajo final fue aprobado como ponencia en el congreso de la Sociedad Latinoamericana de Estudios Sociales de la Ciencia (ESOCITE) de 2008 en Buenos Aires, donde pude participar en un coloquio doctoral dirigido por Arie Rip de la Universidad de Twente (Países Bajos), a quien admiraba por sus trabajos sobre la república de la ciencia (Rip, 1994) y la organización institucional de la ciencia (Rip y Nederhof, 1986). Allí entendí que tenía algo valioso entre manos. El profesor Dominique Vinck, de la Universidad de Grenoble (Francia) quien citaba mi libro con Chavarro de 2006 sobre redes (Pérez y Vinck, 2009), me animó a explorar la colaboración científica desde el punto de vista de los estudios organizacionales, ya que consideraba que podría dar un aporte a la teoría de los estudios sociales de la ciencia y la tecnología.

El segundo semestre de 2008 consistió en la preparación de los exámenes comprensivos. La encomienda era ser suficientes en los debates teóricos de la administración y hacer un seminario de investigación. Me enfoqué en la responsabilidad social con Roberto Gutiérrez, elaborando una investigación sobre el impacto social de la ciencia, para el caso de la enfermedad de Chagas (Orozco y Chavarro, 2011; Orozco, Chavarro y Buitrago, 2012).

7 Ver Semana Educación, n. 15, abril de 2016, pp. 46-47. 
Para 2008 empezó a conocerse que la estrategia de formación doctoral de la UNAL estaba naufragando, ya que sus promediados 50 estudiantes no tenían director de tesis en su malogrado convenio con una universidad de Francia. Se escuchaban historias sobre tres profesores que trabajaban asuntos de epistemología de la administración en el seno de Ascolfa, y que estaban a la deriva en Francia buscando alternativas para terminar su doctorado en administración. Sabía también que en EAFIT el enfoque cualitativo estaba provocando retos enormes para la investigación empírica doctoral. Yo veía de forma más cómoda mi situación en el doctorado de Uniandes, enfocado en el modelo cuantitativo de investigación.

Sin embargo, también supe del doctorado de la Universidad EAN, en asocio con la Universidad Antonio de Nebrija de España. Conocía a uno de sus primeros doctorandos y veía que no tenía tanto sufrimiento, no tenía un examen comprensivo, no padecía las largas revisiones de literatura ni el rigor matemático que tenía por obligación en Uniandes. En lo particular pasé el segundo semestre de 2008 leyendo, haciendo escritos y corriendo modelos estadísticos con bases de datos, tratando de satisfacer las inagotables exigencias de mis maestros, así como prepararme para el examen comprensivo de fin de año. El examen sólo lo aprobamos condicionalmente tres estudiantes, con el deber de que en el primer semestre de 2009 presentáramos un artículo de habilitación. Entendía que una facultad de administración triple corona ${ }^{8}$ como la de Uniandes, solo graduaría a una parte de la primera promoción, como una señal al mercado de que su programa es exigente y de alta calidad. Ciertamente de 12 estudiantes admitidos nos graduamos seis: tres en finanzas y tres en estudios organizacionales.

\subsection{La tesis doctoral}

En 2009 ya había superado el examen comprensivo y debía afrontar el reto de hacer una tesis que aportara nuevo conocimiento en el plano teórico. Marco Palacio, ex rector de la UNAL, nos dio una conferencia sobre la investigación doctoral, y me indicó que debía hacer una tesis sobre un tema y un campo en el que tuviera experiencia para encontrar de mejor forma un aporte de nuevo conocimiento, y sentía que debería ser capaz de ubicar un espacio para proponer algo nuevo entre los estudios organizacionales y las teorías de la sociología de la ciencia. Sabía por Villaveces que la tesis no era aplicar simplemente una teoría o un modelo para evidenciar con el caso colombiano su validez o no, cosa que

8 Cuenta con las tres acreditaciones internacionales para escuelas de administración más importantes: AACSB, AMBA y EQUIS. 
se hacía en las maestrías, sino que era necesario ubicar un aporte teórico, una explicación novedosa a un fenómeno proponiendo nuevas relaciones de conceptos y variables.

Dado mi trabajo previo con Eduardo Wills en su seminario, y mi experiencia en ESOCITE, le pedí que dirigiera mi tesis doctoral a partir de los avances sobre el artículo de habilitación que extendía el trabajo final de su seminario. Wills me urgía descifrar un problema de investigación que fuera relevante en el campo de los estudios organizacionales. El trabajo presentado en ESOCITE era la evaluación empírica de la relación entre capital social, desde la teoría de los vacíos estructurales de Burt (2000), con la producción científica. Había construido para ello las redes de coautoría de los grupos de investigación de Uniandes con mucho esfuerzo en un lapso de un año. Sin embargo, este trabajo no aportaba nada nuevo a la teoría, ya que las explicaciones estaban dadas en la literatura. Solo podía mostrar lo que pasaba para el caso particular que analizaba.

Tenía claro que un aporte a la teoría busca un por qué, una explicación, pero sentía que ya todo estaba explicado. Intentaba buscar en la literatura un vacío, algo que no se hubiera investigado, una relación entre variables no contempladas, pero siempre encontraba artículos, y escuelas de pensamiento, que atendían mis inquietudes. Trabajé con el enfoque multinivel para tratar de ampliar la dicotomía del capital social de Burt (2000) entre redes cerradas y abiertas, pero encontraba muchos artículos sobre ese asunto (Lazega et al., $2006,2008)$. El estudio del capital social en la colaboración científica ya contaba con numerosos aportes y explicaciones, y no hallaba por donde formular un problema de investigación que aportara a la teoría. Me pasé entonces al debate entre la teoría de los costos de transacción y la teoría de dependencia de recursos, pero también encontré literatura que utilizaba estos marcos de referencia para estudiar la colaboración científica. Para el primer semestre de 2010 no tenía nada concreto para presentar a mi director de tesis.

Empecé a elaborar una elucubración sobre la relación entre asuntos de la teoría institucional y la teoría de ecología de poblaciones, a partir de mi reflexión sobre la importancia de las diferencias entre investigadores, y entre sus organizaciones en la producción de nuevo conocimiento científico en el marco de las discusiones de la sociología de la ciencia de Merton $(1968,1977)$ y Bourdieu $(1973,2003)$. Hoy entiendo que esa orientación estaba marcada por mi experiencia en el 0 CyT, donde participé en redes diversas y heterogéneas.

Me había inspirado en el libro de Blau (1977) sobre diversidad y heterogeneidad, el de Adler et al. (2004) sobre la colaboración en administración, el artículo de Barjak y Robinson, (2007) y el libro de Shrum, Genuth y Chompalov (2007) sobre la colaboración científica 
y Harrison y Klein (2007) sobre la definición de la diversidad organizacional. En estos asuntos no había encontrado un estudio empírico que aclarara la diferencia multinivel entre diversidad y heterogeneidad en las redes de colaboración científica, y sentía que por ese camino podría desarrollar mi contribución a la teoría.

Para construir la información sobre la diversidad y la heterogeneidad debía analizar cada artículo, buscar información de cada autor, encontrar las organizaciones que financiaban o participaban en las investigaciones y armar una base de datos, en un problema metodológico multinivel que agregara investigadores con grupos de coautoría y con organizaciones a las que pertenecían, incluyendo también las que aparecían financiando o cooperando en los artículos. Era un trabajo maratónico, nada parecido al de Acedo et al. (2006) sobre las redes de coautoría en administración. Decidí hacer un piloto empírico con los grupos de investigación en administración en Colombia, pero a Wills le pareció irrelevante ya que me sugería la importancia de hacer comparaciones internacionales. Tenía razón. Entonces le propuse trabajar con las escuelas de administración de América Latina escalafonadas por AméricaEconomía. La muestra apenas alcanzaba algo más de 50 escuelas, lo que Wills consideraba poco representativo para un estudio empírico. Sin embargo, yo creía que con esa muestra podría dar un aporte relevante para lo que sabemos sobre el campo de la investigación en administración desde la colaboración científica en redes diversas y heterogéneas. De una parte, había encontrado una publicación del profesor argentino Ernesto Gantman que evaluaba una muestra de escuelas escalafonadas por América Economía para ver los determinantes en la producción científica internacional en administración (Gantman, 2008). De otra parte, la evaluación de las diferencias entre autores y organizaciones no había sido trabajada en la literatura de los estudios organizacionales y de la colaboración científica como lo estaba proponiendo.

En el segundo semestre de 2010 ya no contaba con financiación de Colciencias ${ }^{9}$ y la nueva administración de Uniandes había suprimido los apoyos financieros a los estudiantes. Por ello me vinculé como profesor de cátedra en el Colegio de Estudios Superiores en Administración (CESA) y en la Universidad Externado de Colombia, y aplacé el semestre.

Mi constante revisión de literatura y mis reflexiones sobre el quehacer de un profesor, me permitieron elaborar una descripción sobre la evolución del entorno institucional y el campo de la investigación en administración a la luz de las escuelas de administración, con la que proponía y justificaba un problema de investigación enmarcado en la muestra

$9 \quad$ Me quedaban los fondos para la pasantía en el exterior. 
que empecé a trabajar con el escalafón de América Economía. Le envié a Gantman mis avances, y sus comentarios y observaciones me animaron a continuar mi investigación. Hoy soy consciente de la importancia que tuvo para mí mostrar mis avances de tesis fuera de mi círculo cotidiano, con autores importantes. Gantman haría la presentación de mi tesis doctoral, enfatizando que el capítulo sobre la historia y evolución de las escuelas de administración era por sí solo un aporte muy valioso al nuevo conocimiento.

Había encontrado muchas cosas sobre las escuelas de administración y su escalafonamiento a partir de los microdatos de mi experimento. Evidenciaba cómo la producción internacional de alguna escuela era realizada casi por completo por sus profesores visitantes (asiáticos y anglosajones particularmente), sobre temas y casos ajenos a Latinoamérica. También encontré como alguna escuela concentraba toda su producción en una revista y descubrí que existía un acuerdo entre la escuela y la revista. Alguna vez compartí estos resultados con el profesor Alejandro Beltrán, sin saber que era el decano de la Facultad de Administración de Empresas del Externado. Hoy creo que esa charla casual fue determinante para que en enero de 2011 me vinculara como profesor de planta.

Después de firmar el contrato me fui con licencia no remunerada a realizar la estancia doctoral en la entonces Faculty of Management and Governance de la Universidad de Twente (hoy Faculty of Behavioural, Management and Social Sciences), en una de las más prestigiosas escuelas sobre los estudios de política científica y tecnológica en el mundo, el STEPS, gracias a mi relación con Gonzalo Ordoñez, externadista, y a quien conocía desde el OCYT (fue su primer director). Ese semestre pude poner a prueba mi problema de investigación en un seminario doctoral de alto rigor. Recibí valiosas contribuciones de Arie Rip, Peter Stegmaier, Stefan Kuhlmann y Gonzalo Ordoñez en particular. Logré formular algo sensato, enmarcado en las teorías organizacionales que venía trabajando, para el campo de las escuelas de administración más reputadas de América Latina. Sin embargo, Wills nunca aceptó mi propuesta de tesis doctoral.

Regresé a Colombia a mediados de 2011 satisfecho por haber demostrado que mi creatividad daba avances a los estudios organizacionales sobre ciencia, tecnología y sociedad. Mi propuesta doctoral, afinada en el STEPS de Twente fue aprobada en dos eventos de investigación internaciona ${ }^{10}$. Demostré entonces que tenía algo valioso y que debía volver al seno de mi grupo de investigación informal con Villaveces, Chavarro, Ruiz y Bonilla.

10 The Phil Days - Sussex University, Reino Unido, y The Atlanta Conference - Georgia Institute of Technology, Estados Unidos. 
Para el segundo semestre de 2011 me fue aprobado un nuevo director de tesis, uno que era por derecho propio mi prócer intelectual, uno con quien había trabajado desde 2002, y con quien siempre permaneceré como su discípulo principal, José Luis Villaveces. Aplazamos el segundo semestre de 2011 para sentar los fundamentos de la tesis y buscamos financiación con un proyecto de consultoría para pagar el semestre de aprobación de la propuesta de tesis y el semestre de defensa de la disertación.

Gracias a la colaboración con Bonilla y Chavarro, pude construir las redes de colaboración con la información bruta que elaboré revisando artículo por artículo, autor por autor y escuela por escuela de la muestra de América Economía para dar cuenta de la diversidad entre coautores y la heterogeneidad entre las organizaciones. Era una información de la que no había encontrado antecedentes en la literatura de la colaboración científica, y con la que podría poner a prueba nuevas hipótesis sobre el campo de la investigación en administración a nivel teórico.

Sustenté mi propuesta de tesis doctoral en mayo de 2012 y recibí importantes críticas constructivas de mis jurados, unas orientadas a cuestionar la selección de teorías y debates teóricos, y otras, las más agudas sobre la metodología estadística. Me sugirieron incorporar redes neuronales y estadísticas no paramétrica que desconocía por completo. Me dediqué a definir un procedimiento estadístico que demostrara las relaciones entre las variables propuestas, inspirado en la epistemología de Feyerabend, esto es, sin seguir una metodología ya establecida, pero que tratara secuencialmente una demostración usando las herramientas sugeridas. Superados los asuntos conceptuales, esto es, un documento valioso desde la revisión de la literatura con un problema de investigación bien formulado para aportar a la teoría, el segundo semestre de 2012 consistió en entender matemáticamente el conjunto de técnicas estadísticas que proponía para explicar las relaciones propuestas. El profesor Villaveces me exigió demostrar con papel y lápiz las lógicas de la artillería estadística que proponía. Volví al sufrimiento de la estadística matemática, pero gracias de nuevo a la colaboración científica con un econometrista, estudiante de la tercera cohorte del doctorado, pude entender mis propuestas estadísticas. De nuevo, entiendo hoy, la fortaleza de los enlaces débiles de Granovetter. Finalmente defendí mi tesis en noviembre de 2012 y obtuve la aprobación anhelada, y la tesis fue publicada tres años después, luego de una evaluación de pares, por la Universidad Externado de Colombia (Orozco, 2015), así como un artículo en revista ISI enfocado en el fenómeno de la heterogeneidad (Orozco y Villaveces, 2015). El doctorado en administración me permitió extender mis capacidades previas en investigación, lo que me valió, ya fungiendo como doctor en administración, ser escogido como profesor del doctorado en administración de 
la Universidad Externado de Colombia que inició en 2015. Un año después fui reconocido como investigador asociado por el sistema ScienTI de Colciencias.

\section{Reflexiones}

La política de los doctorados nacionales ejerce presión no solo para obtener el título y ser parte de los grupos de investigación que inscribe el programa, sino para generar publicaciones y otros resultados de investigación que justifican la condonación del crédito. En esa vía, la financiación de Uniandes exigía para la condonación, la producción de artículos científicos, y ofrecía incentivos para la colaboración científica en los grupos de investigación formalmente registrados en ScienTI Colombia. Sin duda estas condiciones impulsan coercitivamente que el doctorando se forme como investigador capaz de publicar. Sin embargo las dinámicas de la formación doctoral conducen a que el educando aproveche sus redes de relaciones informales, en las que se apoya para mejorar sus posibilidades de crear conocimiento y graduarse, como describe el caso.

La formalidad de los grupos de investigación en Colciencias no valora las dinámicas de la colaboración científica y genera problemas para registrar la producción. En mi caso, pertenecía a un grupo en la Vicerrectoría de Investigaciones de Uniandes y en el doctorado fui vinculado a otro. ¿A qué grupo le pertenecen mis publicaciones? Un doctorando debe vincularse a un grupo del programa, como requisito en Colciencias, pero ¿qué pasa si publica solo o con sus colaboradores externos?

Con el caso se aprecia que el cambio de director de tesis, así como la publicación con coautores fuera del núcleo de profesores del programa, está orientado por una lógica que no está atada a los incentivos económicos. Por ello, entiendo que las redes de colaboración científica no responden solamente a motivaciones económicas y orientaciones institucionalizadas.. Por lo tanto, la idea de que los grupos formales de Colciencias impulsan la producción científica de calidad en administración debe contemplar la importancia de las relaciones y capacidades que no se pueden inscribir en el sistema de ScienTI Colombia (Dávila y Malaver, 2004; Malaver, 2006; Orozco et al., 2013).

Investigaciones sobre las redes de producción científica e innovación proponen que las relaciones pasadas contribuyen a la formación de relaciones futuras (Powell y Grodal, 2005). El caso muestra la importancia de mantener las redes previas para afrontar los retos futuros. El proceso de formación doctoral involucra una serie de retos personales. Al entrar al doctorado las relaciones fuertes se vuelven débiles, ya que el trabajo individual 
y la interacción con los profesores, requieren mucho tiempo. Sin embargo, mantener las relaciones con quienes no solo se ha trabajado, sino con quienes se tiene una relación fuerte en términos de tiempo, intensidad emocional, intimidad y reciprocidad como teoriza Granovetter (1973), resulta determinante para acceder a gran diversidad de relaciones fuera del entorno de referencia de la Facultad y mejorar el desempeño individual.

Autores destacados en los estudios organizacionales como Williamson (2005), indican que el doctorado aporta la capacidad de ser disciplinado, esto es, comportarse como un científico leyendo, pensando y escribiendo permanentemente; ser interdisciplinario, o sea, buscar y estudiar diferentes campos de la ciencia; $y$ tener una mente activa, lo que se traduce en preguntas constantes sobre los fenómenos para aprovechar la carencia de explicaciones en la literatura. Sin embargo, Rousseau (2005) y Staw (2005), describen cómo sus relaciones familiares y personales contribuyeron en sus doctorados, facilitando enfocar problemas de investigación relevantes para la teoría organizacional.

La modesta descripción de mi proceso doctoral indica que la colaboración científica, particularmente diversa, externa a un grupo social de referencia, como muestran trabajos empíricos sobre el capital social en la investigación científica (Oh, Chung y Labianca, 2004; Reagans y Zuckerman, 2001) es un factor que también contribuye a la formación doctoral y la generación de nuevo conocimiento.

Coincido con Mishra (2015, p. 185) sobre la importancia de describir la formación doctoral en administración como un viaje de varias etapas, en el que una reflexión personal contribuye a entender el proceso de propuesta y creación de nuevo conocimiento. Pero quizá más relevante es el llamado de Mishra a que el doctorando aprenda a manejar sus relaciones, no solo profesionales, sino familiares. En el caso que describo omití mi entorno familiar, pero debo decir que fue un asunto muy complejo. No tener tiempo para dedicar a la familia genera problemas y tensiones que se deben sortear.

En varios trabajos cienciométricos se puede leer mi preocupación por el Efecto Mateo propuesto por Merton (1968), (Villaveces y Orozco et al., 2007, 2008, 2009), asunto que extendí en mi tesis doctoral (Orozco, 2015). Entendía que la lógica del campo de la ciencia favorecía a quienes producían aportes originales al conocimiento, y sobre todo, a quienes más producían ciencia de calidad. Invito a los estudiantes doctorales a que busquen en la participación en congresos y en la publicación de artículos la legitimación de su trabajo investigativo. Los doctorados en Europa están exigiendo que las tesis doctorales se fundamenten en artículos científicos publicados, y esa lógica de la institución de la 
ciencia no se puede desconocer. También espero que encuentren apoyo en la colaboración científica para mejorar la capacidad de generar publicaciones que certifiquen la calidad del trabajo para ser verdaderos doctores.

Cierro con una reflexión sobre la formación doctoral en Colombia, que enfrenta un reto importante del que empecé a ser consciente con los datos de mi tesis doctoral, que indicaban, como otros estudios (Ordoñez-Matamoros, Cozzens y García, 2010) una creciente colaboración internacional como mecanismo para publicar en revistas indexadas en ISI. Las escuelas de administración más reputadas de América Latina tienen una presión institucional no solo en la política de investigación de los países y sus universidades, sino por los escalafones y las acreditaciones internacionales (Orozco, 2015), que los obliga a crear más diversidad a través de la incorporación de investigadores extranjeros, especialmente anglosajones, que aportan redes internacionales que facilitan la publicación internacional.

Sé de dos colegas, uno con doctorado nacional, y otro formado en una universidad de Inglaterra, que aplicaron a convocatorias de dos escuelas de administración muy reputadas en Colombia, encontrando que sus políticas están orientadas a contratar investigadores, especialmente anglosajones. Las políticas nacionales de investigación han promovido la formación de doctores en el país y en el exterior, pero veo que las plazas de planta se están cerrando, y la capacidad de incorporación de doctores en administración se está constriñendo. Quizá por ello Colciencias ha lanzado una convocatoria para investigar la capacidad de absorción de doctores en el SNCTI.

He descrito cómo la formación doctoral en una escuela de administración de élite en América Latina contribuye al desarrollo de la investigación, en la que redes fuertes y débiles se conjugan para generar publicaciones y crear una comunidad científica que trasciende las facultades de administración y las formalidades de los grupos de Colciencias. Sin embargo, es necesario investigar si la formación doctoral en Colombia, cuenta con un mercado atractivo para insertar investigadores nacionales o si se valora más la incorporación de doctores de países del Norte que pueden, en principio, incrementar la producción internacional. Es necesario investigar y reflexionar sobre este fenómeno para construir el futuro de la comunidad científica en administración. 


\section{Referencias}

Acedo, F.; C. Barroso, C. Casanueva, and J. Galán. (2006). Co-authorship in management and organizational studies: An empirical and network analysis. Journal of Management Studies, 43 (5), 957-983.

Adler, N.; A. Shani, and A. Styhre (eds.) (2004). Collaborative research in organizations: enabling change, learning and theory development. New York: Sage.

Barjak, F., and S. Robinson. (2007). International collaboration, mobility and team diversity in the life sciences: Impact on research performance. Social Geography Discussions, 3 (1), 121-157. doi: 10.5194/sgd-3-121-2007.

Blau, P. (1977). Inequality and heterogeneity: A primitive theory of social structure. New York: Free Press.

Bourdieu, P. (1976). Le champ scientifique. Actes de la recherche en sciences sociales, 2 (2-3), 88-104.

Bourdieu, P. (2003). El oficio de científico. Ciencia de la ciencia y reflexividad. Barcelona: Anagrama.

Burt, R. (2000). The network structure of social capital. Research in Organizational Behavior, 22, 345-423.

Calderón, G. y G. Castaño. (2005). La investigación en administración en América Latina: evolución y resultados. Manizales: Universidad Nacional de Colombia.

Calderón, G.; J. Arrubla, L. Gutiérrez, G. Castaño, R. Posada, A. Ruiz, H. Serna y J. Vivares. (2010). La investigación en administración en Colombia. Condiciones para la generación de conocimiento, investigadores, institucionalización y producción científica. Bogotá: Ascolfa.

Cárdenas, J.H. (eds.) (1991). Doctorados: reflexiones para la formulación de políticas en América Latina. Bogotá: TM Editores y Universidad Nacional de Colombia - CID.

Chavarro, D.A.; L.A. Orozco y J.L. Villaveces. (2010). Análisis del perfil de los grupos de referencia del país. En L.A. Orozco (ed.) La investigación en Uniandes. Construcción de una política. Bogotá: Ediciones Uniandes.

Dávila, C. (1980). La crisis de la educación en Administración en Colombia. Revista Eafit Temas Administrativos, 39, 20-35.

Dávila, C. (1986). La investigación en administración: anotaciones sobre la experiencia colombiana y políticas para su promoción a nivel nacional y latinoamericano. Revista Cátedra, 1, 13-27.

Dávila, C. (1989). Grounding management education in local research: A Latin American experience. In J. Davies, M. Easterby-Smith, S. Mann, and M. Tanton (eds.) The challenge to western management development. London: Routledge.

Dávila, C. (1991). The evolution of management education and development in Latin America. Journal of Management Development, 10 (6), 22-31. 
Dávila, C. y F. Malaver. (2004). A propósito del reconocimiento de Colciencias a grupos de investigación en administración. Cuadernos de Administración, 17 (27), 5-9.

Dávila, C., and H. Samper. (1994). Innovative management and organizational development in Latin America. The International Executive, 36 (6), 671-688.

Estébanez, M.; J. Gómez, C. Durlan, B. García, M. Touris, L.A. Orozco, and M. Cruells. (2013). Producción y uso de conocimiento para el desarrollo social. Análisis de redes sociales. Controversias y concurrencias latinoamericanas, 5 (7), 23-44.

Gantman, E. R. (2008). Determinantes de la producción de conocimiento administrativo en escuelas de negocios de América Latina. Visión de Futuro, 9 (1). Retrieved from http://revistacientifica.fce.unam.edu.ar/index.php?option=com_content\&task=view\&id=138\&Itemid=42

Granovetter, M. S. (1973). The strength of weak ties. American Journal of Sociology, 1360-1380. Harrison, D.A., and K.J. Klein. (2007). What's the difference? Diversity constructs as separation, variety, or disparity in organizations. Academy of Management Review, 32 (4), 1199-1228.

Husserl, E. (1949). Ideas relativas a una fenomenología pura y una filosofía fenomenológica. Trad. J. Gaos, México: FCE.

Laverty, S. M. (2003). Hermeneutic phenomenology and phenomenology: A comparison of historical and methodological considerations. International Journal of Qualitative Methods, 2 (3), 21-35.

Lazega, E., L. Mounier, M. Jourda, and R. Stofer. (2006). Organizational vs. personal social capital in scientists' performance: A multi-level network study of elite French cancer researchers, 1996-1998. Scientometrics, 67 (1), 27-44.

Lazega, E.; M. Jourda, L. Mounier, and R. Stofer. (2008). Catching up with big fish in the big pond? Multi-level network analysis through linked design. Social Networks, 30 (2), 159176. doi: 10.1016/j.socnet.2008.02.001.

Malaver, F. (2000). La investigación en gestión empresarial. Academia Revista Latinoamericana de Administración, 23, 62-77.

Malaver, F. (2006). El despegue de la investigación colombiana en administración: análisis de sus avances en el periodo 2000-2006. Cuadernos de Administración, 19 (32), 71-109.

Merton, R. (1968). The Matthew effect in science. Science, 159 (3810), 56-63. Retrieved from http://www.garfield.library.upenn.edu/merton/matthew1.pdf.

Merton, R. (1977). La sociología de la ciencia: investigaciones teóricas y empíricas. Madrid: Alianza Editorial.

Mishra, P. (2015). Doctoral journey of a management scholar: A viewpoint. Vision: The Journal of Business Perspective, 19 (3), 185-188.

ОСуТ (2009). Indicadores de Ciencia y Tecnología. Colombia 2009. Bogotá: Observatorio Colombiano de Ciencia y Tecnología. 
Oh, H.; M. Chung, and G. Labianca. (2004). Group social capital and group effectiveness: The role of informal socializing ties. Academy of Management Journal, 47 (6), 860-875.

Ordoñez-Matamoros, G.; S. Cozzens, and M. Garcia. (2010). International co-authorship and research team performance in Colombia. Review of Policy Research, 27 (4), 415-431.

Orozco, L.A. (2009). The practice of management. Innovar Journal, 19 (33), 142-144.

Orozco, L.A. (2015). Diversidad y heterogeneidad en redes de colaboración científica. Un estudio de las escuelas de administración de América Latina. Bogotá: Universidad Externado de Colombia.

Orozco, L.A. y D.A. Chavarro. (2005). De la investigación al mercado. Un acercamiento a la medición del impacto de las heliconias colombianas. Estudios Gerenciales, 96, 107-126.

Orozco, L.A. y D.A. Chavarro. (2006). De la historia y sociología de la ciencia a indicadores y redes sociales. Análisis de la biotecnología para el estudio de comunidades científicas en el marco de los programas nacionales de Ciencia y Tecnología. Bogotá: OCyT.

Orozco, L.A. y D.A. Chavarro. (2008). Universidad y emprendimiento. Hallazgos revista de investigaciones, 10, 65-97.

Orozco, L.A. y D.A. Chavarro. (2010). La ciencia como institución. Revista de Estudios Sociales, 37, 143-162.

Orozco, L.A y D.A. Chavarro. (2011). El programa de investigación del CIMPAT - Centro de investigaciones en microbiología y parasitología tropical de la Universidad de los Andes. Bogotá: Ediciones Uniandes.

Orozco, L.A.; C.F. Ruiz, R. Bonilla y D.A. Chavarro. (2013). Los grupos de investigación en Colombia. Sus prácticas, su reconocimiento y su legitimidad. En M. Salazar y L. Fog (eds.) Colciencias cuarenta años: entre la legitimidad, la normatividad y la práctica. Bogotá: OCyT.

Orozco, L.A.; D.A. Chavarro y C.F. Ruiz. (2010). Los departamentos de I+D y la innovación en la industria manufacturera de Colombia: análisis comparativo desde el comportamiento organizacional. Innovar Journal, 20 (37), 103-117.

Orozco, L.A.; D.A. Chavarro y C.F. Ruiz. (2012). Redes e híbridos como formas organizacionales de gobernanza en la biotecnología en Colombia. I Encuentro de investigadores en Administración. Bogotá: Universidad Externado de Colombia y Universidad del Valle.

Orozco, L.A.; D.A. Chavarro y H. Rivera. (2007). Estrategia y gestión del conocimiento. Revista Universidad \& Empresa, 13, 37-58.

Orozco, L.A.; J. Buitrago y D.A. Chavarro. (2012). Biotecnología y valor social: el emprendimiento científico del CIMPAT para enfrentar la enfermedad de Chagas en Colombia. En E. Hodson (ed.) Biotecnologías e innovación. El compromiso social de la ciencia. Editorial Javegraf.

Orozco, L.A; D.A. Chavarro, D. Olaya and J. Villaveces. (2007). Methodology for measuring the socio-economic impacts of biotechnology: A case study of potatoes in Colombia. Research Evaluation, 16 (2), 107-122. 
Pérez, C. y D. Vinck. (2009). Redes sociotécnicas de cogestión de conocimiento en nanotecnologías en Colombia: ¿entre la visibilidad internacional y la apropiación local? Redes, 15 (29), 113-137.

Powell, W., and S. Grodal. (2005). Networks of innovators. The Oxford handbook of innovation. Oxford UK: 0xford University Press.

Reagans, R., and E. Zuckerman. (2001). Networks, diversity, and productivity: The social capital of corporate R\&D teams. Organization Science, 12 (4), 502-517.

Rip, A. (1994). The republic of science in the 1990s. Higher Education, 28 (1), 3-23. doi: 10.1007/ BF01383569.

Rip, A., and A.J. Nederhof. (1986). Between dirigisme and laissez-faire: Effects of implementing the science policy priority for biotechnology in The Netherlands. Research Policy, 15, 253-268.

Ruiz, C.F.; R. Bonilla, D.A. Chavarro, L.A. Orozco, R. Zarama and X. Polanco. (2010). Efficiency measurement of research groups using data envelopment analysis and bayesian networks. Scientometrics, 83 (3), 711-721.

Schuler, I. y L. A. Orozco. (2007). Managing agricultural biotechnology in Colombia. Electronic Journal of Biotechnology, 3 (10), 1-20.

Scott, R. W. (2001). Institutions and Organizations. London: Thousand Oaks, CA, Sage Publications.

Shrum, W.; J. Genuth, and I. Chompalov. (2007). Structures of Scientific Collaboration. Cambridge MA: MIT Press.

Villaveces, J.L. (1991). Los programas doctorales, algunas reflexiones para su iniciación. En J.H. Cárdenas (ed.) Doctorados: Reflexiones para la formulación de políticas en América Latina (pp. 307-322). TM Editores, Universidad Nacional de Colombia - CID.

Villaveces, J.L. (2005). Redes tecnoeconómicas entre productores y usuarios de conocimiento. En M. Albornoz y D. Ratto (eds.) Indicadores de ciencia y tecnología en Iberoamérica, Agenda 2005. Buenos Aires: Red Iberoamericana de Indicadores de Ciencia y Tecnología - RICYT.

Villaveces, J.L. y L.A. Orozco (2015). Colombia. En S. Barro (coord.) La transferencia de I+D, la innovación y el emprendimiento en las universidades. Educación superior en Iberoamérica - Informe 2015. Santiago de Chile: CINDA, RedEmprendia y Universia.

Villaveces, J.L.; L.A. Orozco, D. L. Olaya, D. Chavarro y E. Suárez. (2005). ¿Cómo medir el impacto de las políticas de ciencia y tecnología? Revista Ibero Americana de Ciencia, Tecnología y Sociedad, 2 (4), 125-146.

Villaveces, J.L.; L.A. Orozco, C.F. Ruiz, D.A. Chavarro, E.J. Llanos, A.E. Silva y B. Herrera. (2007). La investigación en Uniandes. Una aproximación desde la cienciometría. C.F. Ruiz, D.A. Chavarro y L.A. Orozco (eds.) Bogotá: Ediciones Uniandes. 
Villaveces, J.L.; L.A. Orozco, C.F. Ruiz, D.A. Chavarro, E.J. Llanos, A.E. Silva, V. Bucheli y S. Daza (2008). La investigación en Uniandes. Elementos para una política. L.A. Orozco (ed.) Bogotá: Ediciones Uniandes.

Villaveces, J.L.; L.A. Orozco, D.A. Chavarro, C.F. Ruiz, R. Bonilla, V. Bucheli, R. Zarama, L. Delgado. (2010). La investigación en Uniandes. Construcción de una política. L.A. Orozco (ed.) Bogotá: Ediciones Uniandes. 
